\title{
Tratamento cirúrgico da "síndrome do roubo" em acesso vascular para hemodiálise com revascularização distal e ligadura arterial
}

\author{
Surgical treatment of "steal syndrome" at hemodialysis vascular access \\ with revascularization and distal artery ligation \\ Fábio Linardi' ${ }^{1}$ Felipe de Francisco Linardi², José Luis Bevilacqua³, \\ José Francisco M oron Morad ${ }^{4}$, José Augusto C osta 5
}

\section{Resumo}

O bjetivo: 0 propósito do trabalho é apresentar o resultado do tratamento da "síndrome do roubo", sintomática em acesso vascular para hemodiálise, utilizando a técnica da revascularização distal com ligadura arterial.

Pacientes e métodos: No período de dezembro de 2003 a novembro de 2004, quatro pacientes que apresentaram síndrome do roubo sintomática em acesso vascular para hemodiálise foram submetidos à revascularização distal com ligadura arterial e acompanhados até dezembro de 2005. O s quatro pacientes apresentavam dor de repouso, e dois casos evoluíram para lesão trófica.

Resultados: Todos os casos apresentaram regressão dos sintomas, com cicatrização das lesões tróficas e manutenção do acesso vascular, que continuou sendo utilizado para real ização de sessões de hemodiálise.

Conclusões: Concluímos que, atual mente, o tratamento de escoIha da síndrome do roubo sintomática é a revascularização distal com ligadura arterial, pois, além de tratar a isquemia do membro, mantém 0 acesso vascular funcionante.

Palavras-chave: Acesso vascular, síndrome do roubo, hemodiálise, fístula arteriovenosa.

\begin{abstract}
0 bjective: The authors report results for the surgical treatment of ischemic "steal syndrome" resulting from hemodialysis vascular access with distal revascularization-interval ligation procedure.

Methods: From D ecember 2003 through N ovember 2004, four patients with "steal syndrome" resulting from hemodialysis vascular access were submitted to distal revascularization-interval ligation and followed until December 2005. All four patients had rest pain and two developed ulcerations.

Results: All patients presented regression of symptoms, healing of ulcerations, and continued to use the same vascular access for hemodialysis.

Conclusion: The authors conclude that the distal revascularization-interval ligation procedure is the treatment of choice for ischemic "steal syndrome", since it effectively treats the ischemic symptoms and maintains a functioning vascular access.
\end{abstract}

Key words: Vascular access, "steal syndrome", hemodialysis, arteriovenous fistula.

\footnotetext{
1. Professor assistente, Doutor, Departamento de Cirurgia, Centro de Ciências Médicas e Biológicas, Pontifícia Universidade Católica de São Paulo (PUCSP), Sorocaba, SP.

2. Acadêmico de Medicina, Faculdade de Medicina de Bragança Paulista, Universidade São Francisco, Bragança Paulista, SP

3. Nefrologista, Unidade de Diálise, Hospital Evangélico de Sorocaba, Sorocaba, SP.

4. Assistente, Mestre, Departamento de Morfologia, Centro de Ciências Médicas e Biológicas, PUCSP, Sorocaba, SP

5. Professor titular, Departamento de Cirurgia, Centro de Ciências Médicas e Biológicas, PUCSP, Sorocaba, SP.

Trabalho desenvolvido no Centro de Ciências Médicas e Biológicas, Faculdade de Ciências Médicas, PUCSP, Sorocaba, SP, e no Instituto de Hemodiálise de Sorocaba, SP.

Artigo submetido em 29.03.06, aceito em 19.05.06.
} 
0 acesso vascular para hemodiáliseéconsiderado o "calcanhar de Aquiles" da hemodiálise, sendo a causa mais freqüente de internação dos pacientes renais crônicos em tratamento dialítico ${ }^{1}$.

A complicação mais freqüente dos acessos para hemodiálise é a trombose, seguida do aneurisma e pseudo-aneurisma2.

A "síndrome do roubo" sintomática acontece em 1 a $8 \%{ }^{2}$ dosacessos, sendo seusfatores preditivos a fístula arteriovenosa (FAV) que utiliza a artéria braquial como doadora, sexo feminino, idademaior de60 anos, diabetes e doença vascular periférica ${ }^{2,3}$.

O s tratamentos propostos para correção da síndromedo roubo, como abandagem, quevisa diminuir aluz da artéria doadora eautilização deprótesescônicas, não apresentam resultado satisfatório ${ }^{4-6}$. A ligadura daFAV apresenta excelente resultado em relação à isquemia, porém a perda do acesso é inerente, e a construção de novo acesso e/ou a passagem de cateter central se faz necessária?.

Em 1988, Schanzer et al. ${ }^{3}$ descreveram a técnica de revascularização distal com ligadura arterial - distal revascularization-interval ligation (DRILL) - em três casos com excelente resultado. A técnica descrita pelo autor consiste em interposição de substituto arterial com tomada proximal na artéria braquial, $5 \mathrm{~cm}$ antesda anastomose da FAV , tomada distal próximo da bifurcação da artéria braquial eligadura da artéria braquial logo abaixo da anastomose da FAV, com a finalidade de se evitar o fluxo reverso distal.

0 objetivo destetrabalho éapresentar a experiência pessoal com a técnica do D RILL, empregada em quatro casos de isquemia grave induzida pela síndrome do roubo.

\section{M étodo}

No período de dezembro de 2003 a novembro de 2004, a síndrome do roubo sintomática ocorreu em quatro das $155 \mathrm{FAV}$ construídas no período $(2,5 \%)$.

0 diagnóstico dasíndrome do roubo foi eminentemente clínico, baseado na anamnese exame clínico. Em todos os casos, os sintomas principais foram: dor isquêmica de repouso com piora durante as sessões de hemodiálise e, em dois casos, dor de repouso elesão trófica, caracterizada por úlcera de 2 $\mathrm{cm}$ de diâmetro, fundo necrótico e extremamente dolorosa (Figura 1).

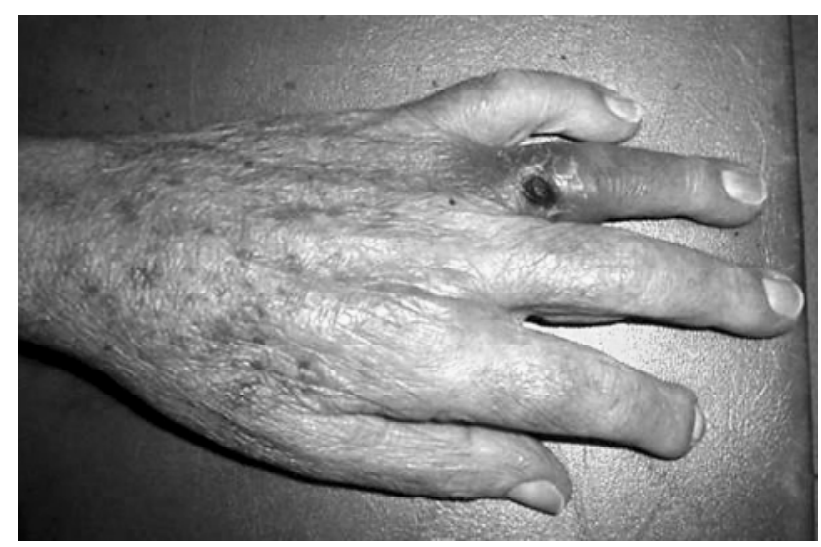

Figura 1 - Úlcera isquêmica

Para avaliação do leito distal, foi utilizada a manobra de compressão da FAV com comprovação do aparecimento do pulso radial.

D ois pacientes eram do sexo feminino, e a idade média foi de 63 anos, variando entre 57 e 68 anos.

A doençaqueocasionou a insuficiência renal crônica foi o diabetes melito em dois casos, mieloma múltiplo no terceiro caso e glomerulonefrite crônica no quarto. As doenças associadas foram: hipertensão arterial, em quatro pacientes; e gota úrica, diabetes melito, insuficiência coronariana e vasculopatia periférica em um. 0 tempo médio de aparecimento dos sintomas, após a construção da FAV, foi de 7 meses, variando de 2 a 21 meses.

T odas as quatro FAV foram proximais, sendo uma braquiobasílica superficializada em membro superior esquerdo, uma braquiocefálica em membro superior esquerdo e duas braquiocefálicas em membro superior direito.

0 tempo médio entre a realização da $F A V$ e a cirurgia do D RILL foi de 12 meses, variando de 4 a 31 meses.

Em relação aos aspectos técnicos das revascularizações, os substitutos utilizados foram a veia baślica em três casos e a veia safena em um caso. A ligadura da artéria braquial abaixo da anastomose foi realizada em um caso, enos demais foi feita apenas a interposição de veia (Figuras 2 e 3 ).

0 s resultados foram avaliados com base em exame clínico, levando em consideração a evolução dos sintomas, e no exame das extremidades, avaliando temperatura, coloração, perfusão e presença dos pulsos distais. 


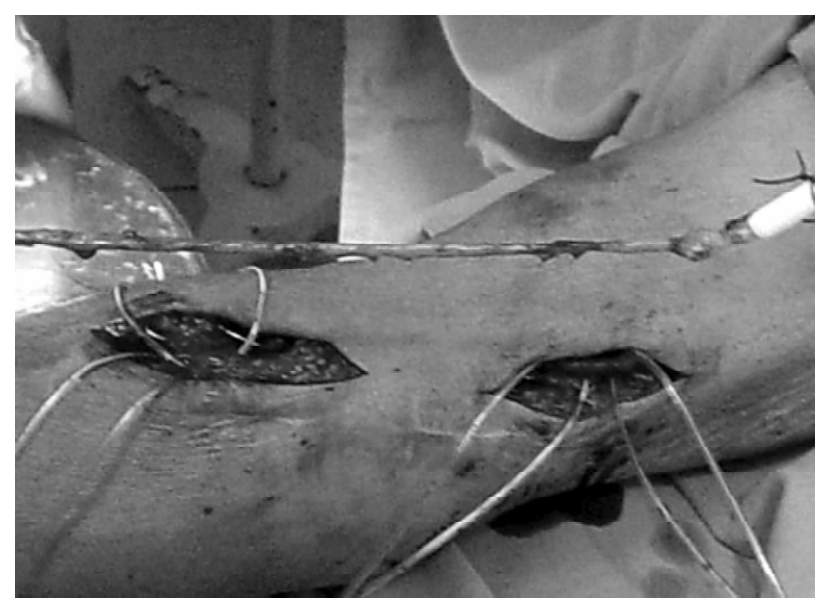

Figura 2 - D issecção proximal e distal e veia substituta

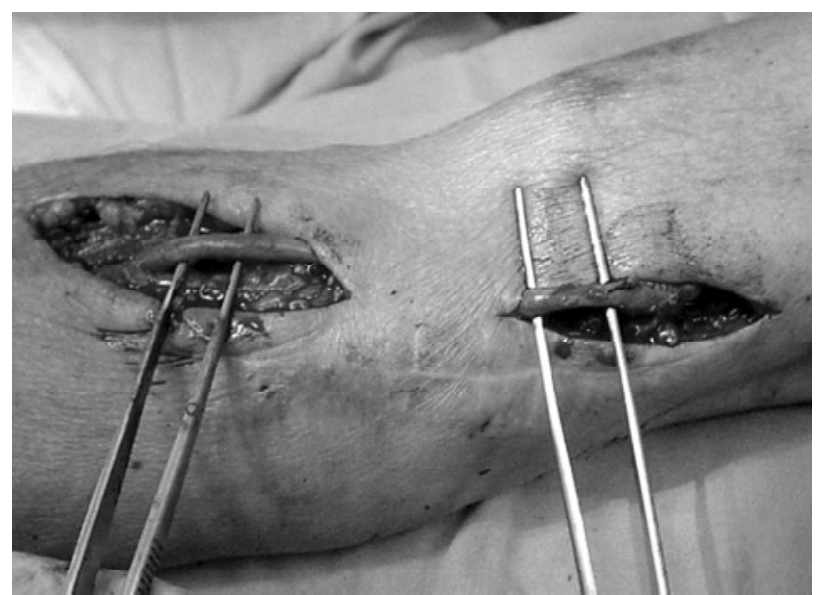

Figura 3 - D erivação

0 tempo de seguimento foi de 24 meses - de dezembro de 2003, quando se realizou a primeira cirurgia, até dezembro de 2005, quando se findou 0 estudo. Todos os casosforam acompanhados atéo final do estudo.

Esta pesquisa foi aprovada pelo Comitê de Ética em Pesquisa do C entro de Ciências M édicas e Biológicas (CCM B) da Faculdade de C iências M édicas da PU C-SP.

\section{Resultados}

O s dois primeiros casos apresentavam dor de repouso, com piora durante as sessões de hemodiálise, e não evoluíram para lesão trófica. N esses dois casos, foi realizada apenas a revascularização distal, com interposição de segmento de veia com anastomose proximal na artéria braquial acima da anastomose da FAV e anastomose distal na bifurcação da artéria braquial. O s dois casos evoluíram com regressão total dos sintomas e aparecimento de pulsos distais.

O terceiro caso evoluiu para lesão trófica caracterizada por duas úlceras isquêmicas com fundo necrótico em falange distal do quarto pododáctilo da mão direita e foi submetido à mesma cirurgia dos casos anteriores. Apresentou cicatrização da lesão e ausência de sintomas, porém manteve a mão com palidez, frialdade e ausência de pulsos distais até o final do estudo. 
0 quarto pacienteapresentava lesão tróficacaracterizada por úlcera de $2 \mathrm{~cm}$ de diâmetro com fundo necrótico, localizada no quarto pododáctilo da mão esquerda, e também foi submetido à mesma cirurgia anterior. Apresentou evolução desfavorável, com manutenção do quadro de isquemia grave, sem nenhum sinal de melhora. Após 1 semana, foi submetido à ligadura da artéria braquial, conforme descrito na técnica original, com regressão total dos sintomas, reaparecimento dos pulsos distais e cicatrização da lesão trófica (Figura 4).

T odos os casos, após revisão dos procedimentos, evoluíram com ausência de sintomas isquêmicos e ainda se mantiveram em programa de hemodiálise utilizando a mesma FAV inicial atéo final do estudo.

\section{D iscussão}

A fisiopatologia do fenômeno do roubo em FAV já foi discutida por Barnes em 19807 0 sistema de baixa pressão presente no lado venoso daFAV induz o fluxo reverso na porção distal da artéria abaixo da anastomose da FAV. Essa alteração da direção do fluxo é que caracteriza o roubo ${ }^{8}$.

Estudoshemodinâmicos ${ }^{9,10}$ constatam que o fluxo retrógrado nas $\mathrm{FAV}$ radiocefálicas ocorre em
73,3\% dos casos e, nas FAV com prótese de 5 a 6,5 $\mathrm{mm}$ utilizando a artéria braquial e a veia axilar, em $90,9 \%$ dos casos.

Lazarides et al. ${ }^{11}$, em estudo prospectivo, mensuram o índice de pressão sistólica (pressão sistólica nas artérias distais do membro superior dividida pela pressão sistólica da artéria braquial contralateral) em 69 pacientes antes da realização daFAV edemonstram que $94 \%$ tinham pressão menor que 0,8 . Realizaram novo índice 24 horasapós a construção daFAV edemonstraram índice de 0,55, e todos os pacientes eram assintomáticos. N ova mensuração após 11 meses demonstrou índice de 0,74. Atribuíram a melhora do índice ao aumento da circulação colateral eà vasodilatação distal .

0 s fatores que determinam a presença de isquemia são: a capacidade de uma artéria proximal de entregar sangue a uma FAV, o tamanho e o comprimento da FAV e a adequação das colaterais ${ }^{3}$.

0 roubo sintomático só acontece quando os mecanismos compensatórios são insuficientes e ocorre em 1 a $8 \%$ das FAV 2 .

O s fatores preditivos para o roubo sintomático são: sexo feminino, diabetes melito, doença vascular periférica e as FAV que utilizam a artéria braquial como doadora ${ }^{2,3}$. 0 melhor tratamento para o roubo

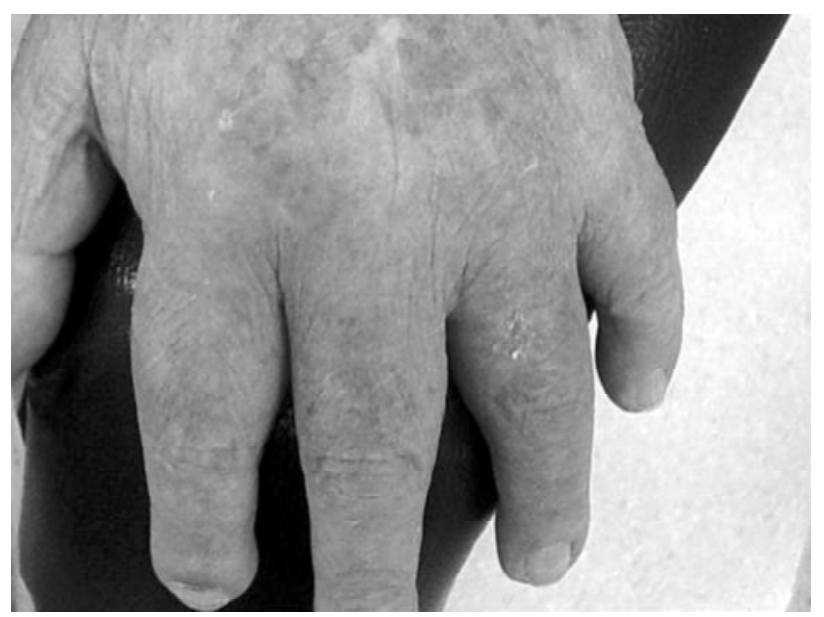

Figura 4 - C Cicatrização 
sintomático é a prevenção, porém não é um método confiável $^{8,12}$.

Além dos fatores preditivos, devese realizar a manobra de Allen, mensurar a pressão sistólica em ambos os membros superiores, ficar atento quando houver assimetria maior que $20 \mathrm{mmH} \mathrm{g}$ e avaliar os pulsos distais antes e após a realização da anastomose. Se houver ausência de pulso distal após a anastomose, deve-se reavaliar a cirurgia ${ }^{3,4,12}$.

0 tratamento da síndrome do roubo sintomática tem dois desafios: resolver a isquemia e manter 0 acesso $^{2}$.

Vários tratamentos têm sido propostos:

- Ligadura do acesso - é o método mais simples, elimina a isquemia em $100 \%$ dos casos, porém a perda do acesso é óbvia.

- Bandagem ou plicatura - consiste em realizar sutura longitudinal na veia ou prótese, logo após a anastomose da FAV, com o objetivo de reduzir 0 diâmetro da FAV entre 20 e $25 \%$.

- U tilização de prótese cônica sugerida por Rosenthal et al. ${ }^{13}$ para prevenção do roubo. As próteses têm 6 mm de diâmetro na anastomose arterial e $4 \mathrm{~mm}$ na anastomose venosa.

As três técnicas propostas acima têm apresentado resultadosmedíocres ${ }^{4-6}$, pois, segundo W ixon et al. ${ }^{14}, 0$ grau de estenose que irá melhorar o roubo está muito próximo do ponto de trombose da FAV.

Em 1988, Schanzer et al. ${ }^{3}$ descreveram a técnica do D RILL, inicialmente utilizada em três pacientes. Em 1992, publicaram novo trabalho com 14 pacientes e obtiveram sucesso em $13^{15}$. Em 1996, $\mathrm{H}$ aimov et al. utilizaram a técnica em 23 pacientes e obtiveram 100\% de resultados ${ }^{8}$. Apesar disso, a técnica obteve pouco reconhecimento ${ }^{2}$.

N os anos subseqüentes, vários autores ${ }^{2,12,16}$ publicaram suas experiências com a técnica, e todos obtiveram excelentes resultados.

0 presente trabalho apresenta dados demográficos como sexo, idade, doença de base, comorbidades e tempo de aparecimento dos sintomas, semelhante aos da literatura.

A não-ligadura da artéria nos três primeiros casos ocorreu por fal ta de experiência pessoal com a técnica e por receio de piorar o quadro isquêmico.
Segundo Barnes ${ }^{12}$, a ligadura da artéria pode ser dispensada na presença de doença arterial oclusiva distal importante, pois dificultaria o fluxo retrógrado arterial, que éa principal característica do roubo sintomático.

\section{Conclusão}

O s resultados obtidos, com resolução da isquemia e manutenção do acesso em todos os casos, até o final deste estudo, permite-nos concluir que a técnica do D RILL éhoje o tratamento de escolha para a síndrome do roubo sintomática.

\section{R eferências}

1. $\mathrm{H}$ akim $\mathrm{R}, \mathrm{H}$ immelfarb J. H emodialysis access failure: a call to action. Kidney Int. 1998;54:1029-40.

2. Knox RC, Berman SS, Hughes JD, G entile AT, M ills JL. $D$ istal revascularization-interval ligation: adurableand effective treatment for ischemic steal syndrome after hemodialysis access. J V asc Surg. 2002;36:250-5; discussion 256.

3. Schanzer $H$, Schwartz $M, H$ arrington $E, H$ aimov M . T reatment of ischemia due to "steal" by arteriovenous fistula with distal artery ligation and revascularization.J V asc Surg. 1988;7:770-3.

4. M orsy $A H$, Kulbaski $M$, Chen $C$, I siklar $H$, Lumsden AB. Incidence and characteristics of patients with hand ischemia after ahemodialysisaccessprocedure.J Surg Res. 1998;74:8-10.

5. D eC aprio JD, Valentine RJ, Kakish H B, Awad R, H agino RT, Clagett GP. Steal syndrome complicating hemodialysis access. Cardiovasc Surg. 1997;5:648-53.

6. Rivers SP, Scher LA, Veith FJ. C orrection of steal syndrome secondary to hemodialysis access fistulas: A simplified quantitative technique. Surgery. 1992;112:593-7.

7. Barnes RW. H emodynamics for the vascular surgeon. Arch Surg. 1980;115:216-23.

8. Haimov $M$, Schanzer $H$, Skladani $M$. Pathogenesis and management of upper-extremity ischemia following angioaccess surgery. Blood Purif. 1996;14:350-4.

9. K wun $K B$, Schanzer $H$, Finkler $N, H$ aimov $M$, Burrows $L$. $\mathrm{H}$ emodynamic evaluation of angioaccess procedures for hemodialysis. Vasc Surg. 1979;13:170-7.

10. Anderson CB, EtheredgeEE, H arter HR, Codd JE, G raff RJ, $\mathrm{N}$ ewton WT. Blood flow measurements in arteriovenous dialysis fistulas. Surgery. 1977;81:459-61.

11. Lazarides M K, Staramos DN, Panagopoulos GN, T zilalis VD, Eleftheriou GJ, D ayantas JN. Indications for surgical treatment of angioaccess-induced arterial "steal". J Am Coll Surg. 1998;187:422-6.

12. Berman SS, Gentile AT, Glickman MH, et al. Distal revascularization-interval ligation for limb salvage and maintenance of dialysis access in ischemic steal syndrome. J V asc Surg. 1997;26:393-402; discussion 402-4. 
13. Rosental JJ, Bell D D, Gaspar M R, M ovius HJ, Lemire GG. Prevention of high flow problems of arteriovenous grafts. D evelopment of a new tapered graft. Am J Surg. 1980; 140: 231-3.

14. W ixon CL, H ughes J D, M illsJ L. U nderstanding strategiesfor the treatment of ischemic steal syndrome after hemodialysis access. J Am Coll Surg. 2000;191:301-10.

15. Schanzer $H$, Skladany $M, H$ aimov $M$. Treatment of angioaccess-induced ischemia by revascularization. J Vasc Surg. 1992;16:861-4; discussion 864-6.

16. Katz S, K ohl RD. The treatment of hand ischemia by arterial ligation and upper extremity bypass after angioaccess surgery. J Am Coll Surg. 1996,183:239:42.

Correspondência:

Fábio Linardi

Av. São Paulo, 2918, Jd. G onçalves

CEP 18013-004 - Sorocaba, SP

Tel.: (15) 227.1612

Fax: (15) 233.2833

E-mail: flinardi@terra.com.br 